\title{
DÜBLIN
}

Technological University Dublin

ARROW@TU Dublin

Conference Papers

Biomedical Devices and Assistive Technology

Research Group

2011

\section{Blood Vessel Diameter Estimation System Using Active Contours}

Jane Courtney

Technological University Dublin, jane.courtney@tudublin.ie

Ana Tizon

Technological University Dublin, atizon@yahoo.com

Follow this and additional works at: https://arrow.tudublin.ie/biodevcon

Part of the Biomedical Engineering and Bioengineering Commons

\section{Recommended Citation}

Courtney, J., Tizon, A. : Blood Vessel Diameter Estimation System Using Active Contours, Proceedings of the 2011 Irish Machine Vision and Image Processing Conference, Dublin City University, 7-9 September.

This Conference Paper is brought to you for free and open access by the Biomedical Devices and Assistive Technology Research Group at ARROW@TU Dublin. It has been accepted for inclusion in Conference Papers by an authorized administrator of ARROW@TU Dublin. For more information, please contact arrow.admin@tudublin.ie, aisling.coyne@tudublin.ie, gerard.connolly@tudublin.ie.

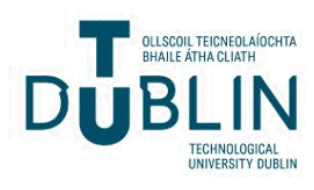


Dublin Institute of Technology

ARROW@DIT

Articles

2011

\section{Blood Vessel Diameter Estimation System Using Active Contours}

Ana Tizon

Jane Courtney

Follow this and additional works at: http://arrow.dit.ie/despart

Part of the Biomedical Commons

This Conference Paper is brought to you for free and open access by ARROW@DIT. It has been accepted for inclusion in Articles by an authorized administrator of ARROW@DIT. For more information, please

contact yvonne.desmond@dit.ie, arrow.admin@dit.ie. 


\title{
Blood Vessel Diameter Estimation System Using Active Contours
}

\author{
Ana Tizon, Jane Courtney \\ School of Electronic \& Communications Engineering \\ Dublin Institute of Technology \\ Dublin, Ireland \\ atizon@yahoo.com
}

\begin{abstract}
The study and analysis of blood vessel geometry has become the basis of medical applications related to early diagnosis and effective monitoring of therapies in vascular diseases. This paper presents a new method to trace the outline of blood vessels from imperfect images and extract useful information about their dimensions in an automated manner. The system consists of a segmentation procedure that uses two Active Contours to detect blood vessel boundaries and a novel approach to measure blood vessel diameters directly as the distance between two points. We have succeeded in designing and implementing an automated, robust, measurement method that is not only accurate (it takes away human error) but also userfriendly and requires very little image pre-processing. The system is tested with a set of grey scale images of blood vessels. Results of all the aspects of the design and implementation are presented along with graphs and images.
\end{abstract}

Keywords- blood vessel diameter; snake; Active Contours; measurement system

\section{INTRODUCTION}

Blood vessels play a very important role in almost every medical condition. Information about blood vessel diameters can be vital in observing the healing process, finding and observing tumor development, diagnosing vascular diseases and learning about the workings of the vascular system. Therefore the study and analysis of vessel geometry features has become the basis of medical applications related to early diagnosis and effective monitoring of therapies in vascular diseases. A substantial amount of research has been done on the development of strategies for accurate measurement of vessel diameters in order to achieve more precise and repeatable diagnostic methods [1]. The critical and challenging task of obtaining precise vessel diameter measurements is dependent on the accuracy of the segmentation method used [2]. Images of veins and arteries can be obtained using intravital fluorescence microscopy. However, such images can be vague and indistinct making it difficult to derive useful information from them [3]. Hence, currently the study of vessel diameter measurements is still an area open for improvements
[4]. In this work, a method will be developed to trace the outline of blood vessels from imperfect images and extract useful information about their dimensions in an automated manner.

Before getting into the detailed description of the system it may be useful to give a simple explanation of the approach taken here. The system design consists of two main parts:

\section{1) Blood Vessel Segmentation}

To obtain two sets of co-linear points (i.e. points that lie on the same line) corresponding to points on the edge of the fragment of blood vessel to be analyzed. This will be achieved using two snakes (Active Contours).

\section{2) Diameter Measurement System}

To design a measurement system that calculates the average distance between the two sets of points. Figure 1 shows the final result of the method applied to a blood vessel image.

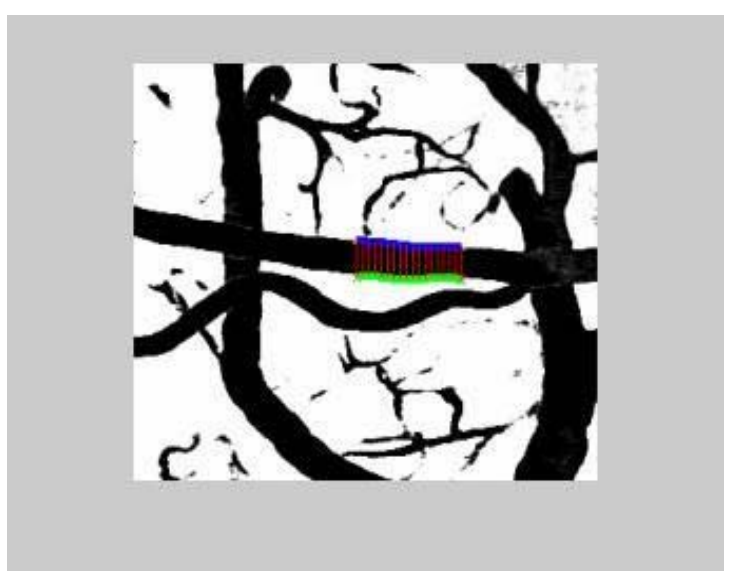

Figure 1. The two snakes and the distance between them 


\section{BLOOD VESSEL SEGMENTATION: SNAKE IMPLEMENTATION}

Deformable models are some of the most popular and successful segmentation methods due to their ability to accurately recover the shape of biological structures in many medical image segmentation applications. Parametric deformable models derive from the Active Contours (snakes) model introduced by Kass [5]. The principle underlying Active Contours is to deform an initial contour towards the boundary of the object to be detected by minimizing an energy function. This energy function is a combination of internal and external energies. Both the Internal Energy (by using geometric shape forces) and the External Energy (by using image forces) restrict contour deformation. Optimizing the weights used in the Internal Energy and selecting adequate image forces (e.g., edges), Active Contour models are used to adapt the curve to the boundaries of objects being segmented.

A simple elastic snake or Active Contour is defined by: a set of $\mathbf{n}$ points (called snaxels), an Internal (elastic) Energy and an External Energy.

Let us consider a snaxel $S_{i}$ :

$$
S_{i}=\left(x_{i}, y_{i}\right) \text { with } i=1 \ldots n
$$

The Total Energy of snaxel $S_{i}$ in its current position is

$$
E_{\text {snaxel }}=\alpha E_{\text {cont }}+\beta E_{\text {curv }}+\gamma E_{\text {image }}
$$

where

$$
\begin{aligned}
& E_{\text {cont }}=\left(x_{i}-x_{i-1}\right)^{2}+\left(y_{i}-y_{i-1}\right)^{2} \\
& E_{\text {curv }}=\left(x_{i-1}-2 x_{i}+x_{i+1}\right)^{2}+\left(y_{i-1}-2 y_{i}+y_{i+1}\right)^{2} \\
& E_{\text {image }}=-\left\|\nabla I\left(x_{i}, y_{i}\right)\right\|
\end{aligned}
$$

are the Internal Energy (Continuity and Curvature) terms and the External Energy (Image) terms respectively and $\alpha, \beta$ and $\gamma$ are weighting parameters chosen to restrict the effect of the corresponding constraints.

The Total Energy of the snake is the sum of the energies of all its snaxels in their current positions and it is given by the following equation:

$$
E_{\text {snake }}=\sum_{i=1}^{n}\left(\alpha E_{\text {cont }}+\beta E_{\text {curv }}+\gamma E_{\text {image }}\right)
$$

For the snake to locate the outline of an object, the following steps must be performed:

1) Initialization of the snake in the vicinity of the object

Once the user has selected two points within the vessel, the next step is to find the equation of the line passing through the two selected points. (Note that every portion of blood vessel selected will be modeled by two snakes, one for each side of the blood vessel).
Given $P_{1}=\left(x_{1}, y_{1}\right)$ and $P_{2}=\left(x_{2}, y_{2}\right)$, the straight line passing through points $P_{1}$ and $P_{2}$ is given by:

$$
y=x\left(\frac{y_{2}-y_{1}}{x_{2}-x_{1}}\right)-x_{1}\left(\frac{y_{2}-y_{1}}{x_{2}-x_{1}}\right)+y_{1}
$$

At this point the "preliminary" set of $\mathbf{n}$ initial points of both snakes can be determined:

$$
\begin{gathered}
y=y_{1}: 4: y_{2} \text { (using Matlab notation) } \\
x=\text { round }\left(\frac{\left(y-y_{2}\right)\left(x_{1}-x_{2}\right)}{\left(y_{1}-y_{2}\right)}+x_{2}\right)
\end{gathered}
$$

The initialization consists of a set of points on a straight line. With regards to the snake Internal Energy, a minimum Energy state is achieved when points lie on a straight line with each snaxel keeping a constant distance from its snaxel neighbor.

When using an open curve, to encourage movement of snaxels towards the edge of the vessel, constraints must be imposed on the end points. The first and the last points of the initial set are brought to the edge of the blood vessel. Since the design involves two snakes, this procedure must be done twice: once for each snake.

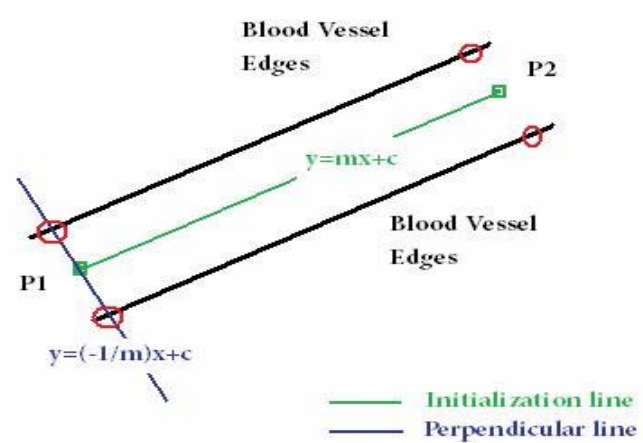

Figure 2. Perpendicular line passing through initial point

\section{For the first snake:}

To determine the direction where these endpoints have to move to reach the closest edge, the method described below is used.

A new line is defined. This new line is perpendicular to the existing initialization line and it passes through the endpoint $\mathrm{P}$ to be moved. Assuming that the calculated perpendicular line is given by:

$$
A x+B y+C=0
$$


The next step is to calculate the distance from this perpendicular line to each of the eight neighboring pixels of point $\mathrm{P}$ as shown in Fig 2.

The distance between a line and a point $P=\left(x_{p}, y_{p}\right)$ is given by:

$$
d=\frac{\left|A x_{P}+B y_{P}+C\right|}{\sqrt{\left(A^{2}+B^{2}\right)}}
$$

The distance from each of the neighboring pixels of point $\mathrm{P}$ to the perpendicular line will be calculated. The edge of the vessel will be then searched in the direction of the neighboring pixel closest to that line. The Sobel operator will be used to find the endpoint edges (in the direction previously calculated). And so a new set of initialization points is determined where all the points remain the same except for the two endpoints that have been moved towards the blood vessel edge.

\section{For the second snake:}

The direction where the endpoints have to move to reach the closest edge for the second snake will be perpendicular to the direction where they moved for the first snake. E.g., if the endpoints of the first snake moved to "up-left" direction then the endpoints of the second snake will move to "down-right" direction and so on. The two new endpoints for each snake are now fixed on the edge of the vessel, i.e. they will not be allowed to move to any other position. This new layout will trigger snaxel movement to positions of minimum Energy.

The initialization method used in this project is a straight line discretized in $\mathbf{n}$ points. The accuracy of the system will depend largely on the number of points used. Figure 3 shows the common initialization line that derives into two initialization lines one for each snake.

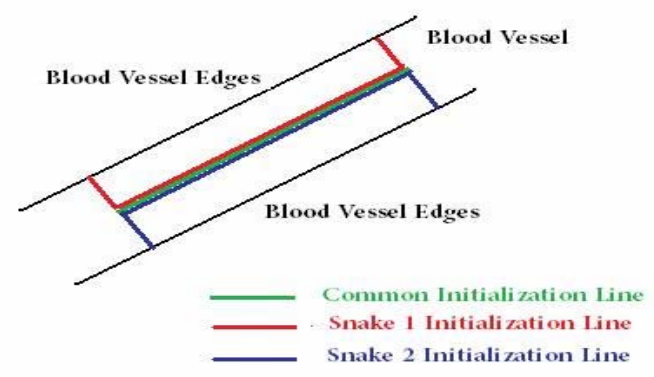

Figure 3. Perpendicular line passing through initial point.

\section{2) Minimization of the Total Energy of the snake}

Since system accuracy can be easily managed (by increasing the number of initial points), to simplify the snake design, the system will have four possible cases regarding snaxels movement. Snaxels will be moved towards the edge of the vessel in the same direction as it was calculated for the initial endpoints in the initialization phase, i.e., the first point of the initialization line will determine the movement direction. With this in mind, here is the explanation on how these 4 possible cases work and their implications in the final snake algorithm. (Figure 4 shows one of the search neighborhood cases). Once the current values of the snaxel's energies are determined (as explained earlier in this section), snaxels must be moved, one by one, to a position of local minima. The "greedy algorithm" [6], the dynamic programming method used in this project, is an iterative method that calculates the movement of each snaxel individually at each iteration.

The algorithm searches a small neighborhood about the current location, recalculating the energies at each neighboring point. The snaxel is then moved to the neighboring point with the lowest energy and the process is continued throughout the snake. After each iteration, the Total Energy of the snake is calculated. While the Total Energy is decreasing the iterations continue. When the Total Energy gets steady (no snaxel movements) then the algorithm ends and the snake is considered to be in its best position.

In this application, the snaxel search neighborhood will be limited to two pixels: "up/down", "right/left", "up-right/downleft" and "up-left/down-right". This will improve computation times without compromising any of the requirements of the system.

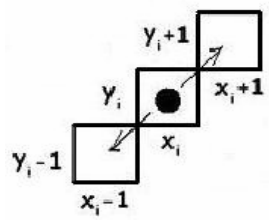

Figure 4. UP-RIGHT/DOWN-LEFT Snaxel search neighbourhood.

\section{3) Multipoints}

After a few initial tests, an overlapping problem was observed as shown in Figure 5. Snaxels that were meant to move to the lower side of the vessel found positions of minimum Energy on the upper side and stayed there. To solve this problem, it was decided to use a "signed" Sobel operator instead. For each case of snaxel movement the Sobel operator is further adapted to have positive and negative components. It will be dealt with as two different Sobel operators: the positive one will be used as the edge attraction term of the External Energy for the first snake, and the negative one will be used as the edge attraction term of the External Energy for the second snake as shown in Figure 6.

Although the results improved considerably, problems were still found in areas where the initialization set of points was close to a second blood vessel, e.g. the snaxels would move towards this new vessel, or under noise conditions. Other methods (e.g. Distance Transform) were tried then to overcome the issue but none of them proved to be totally satisfactory. A well known problem with snakes is that initialization points must be always close to the target object. The final decision to overcome the overlapping issue was to improve the way the snake is initialized. 
The user must select 2 (or more points) inside the vessel to indicate the portion of the vessel where the diameter is to be calculated. Points must not to be selected at places where vessels overlap. Lines joining selected points must always remain within the vessel.

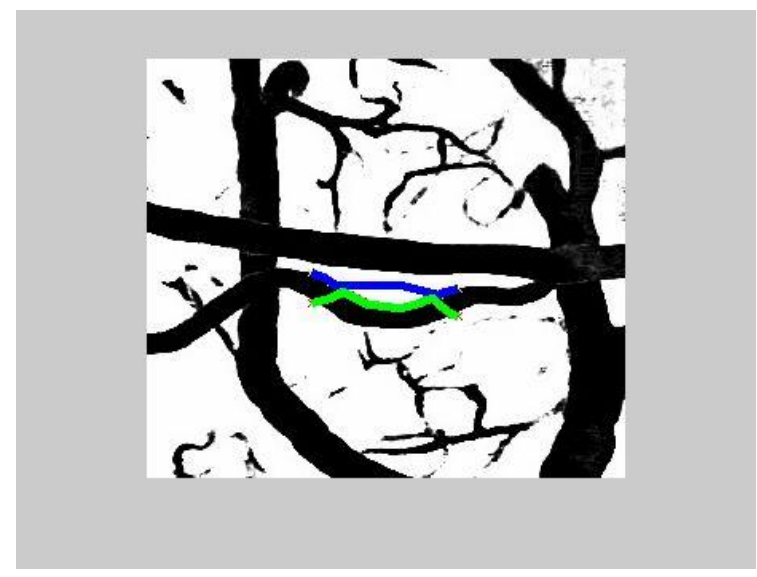

Figure 5. Overlapping Problem.
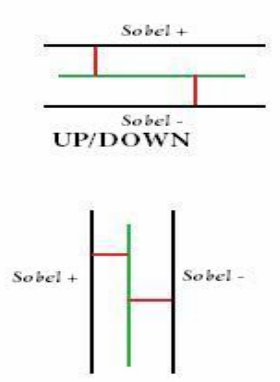

RIGHT/LEFT

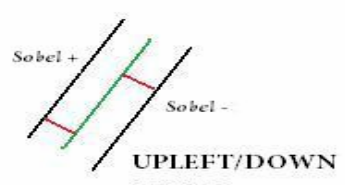

RIGHT

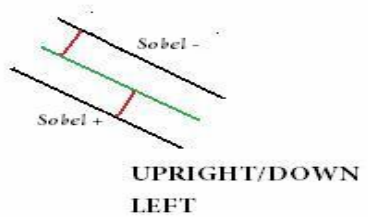

LEFT
Figure 6. Sobel signed operator used in four Snaxel Movement cases.

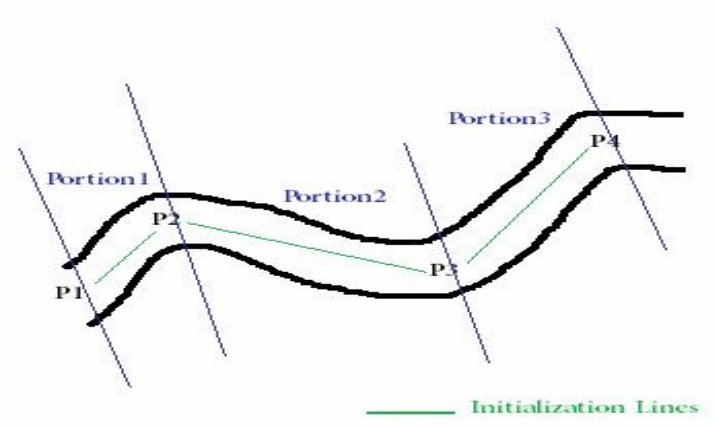

Figure 7. Multipoint selection system principle.

The original system design allowed the user to select only two points inside the vessel. The new method allows selecting as many points as the user wishes (given that the point selection follows the rules set above) is shown in Figure 7. The idea is to treat each portion of vessel (between selected points) independently and then link all the results together. If there is only one portion of vessel the results will be stored in two vectors, one vector for each snake. For additional portions (if there is any) the same method will be applied and the obtained results will be appended to the existing vectors. Most blood vessels will only need two initialization points. The Multipoints method has been mainly designed to cater for more complex vessels shapes (Figure 8).

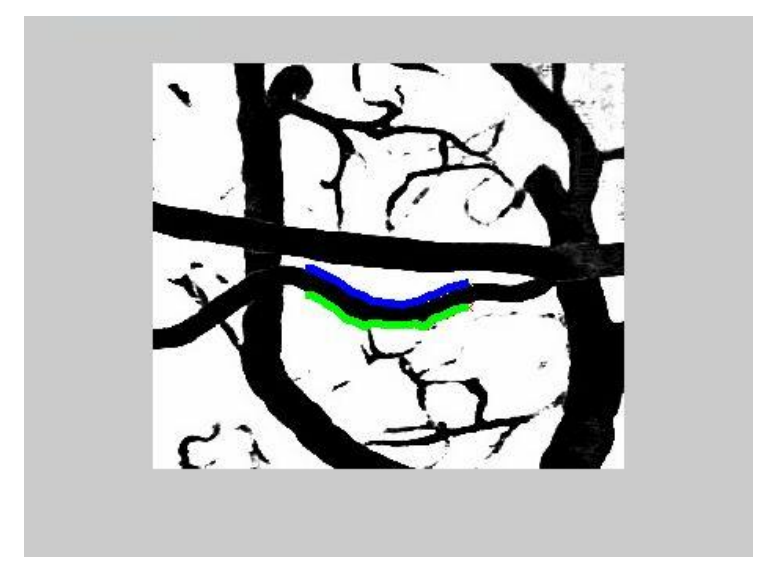

Figure 8. Results obtained with MultiPoints selection method.

\section{DIAMETER MEASUREMENT SYSTEM}

Measurement of the vessel diameter based on Manual Tracing (MT) procedures is dependent on the subjectivity of the reader and the existing tracing procedures that often fail to detect the vessel boundaries accurately. The measurement system proposed in this work it measures vessel diameters directly as the distance between two points. These two points are situated on curves representing the detected boundaries (snake1 and snake2) that are perpendicular to the vessel orientation. Here there is a brief description of the system (illustrated in Figure 9):

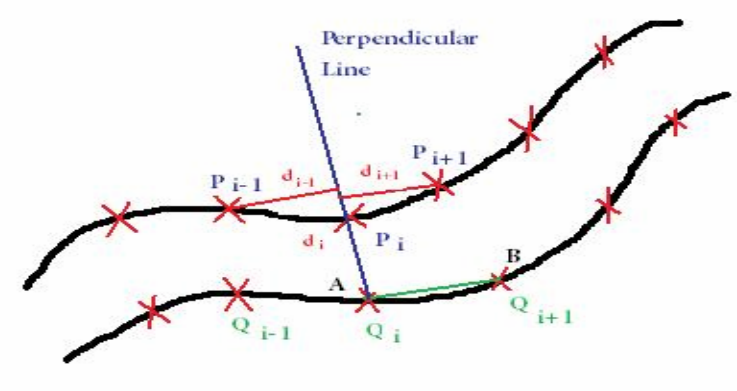

Figure 9. Measurement System: Distance Point Decision.

Two sets of points have been obtained through Blood Vessel Segmentation techniques (Active Contours). The first step is to calculate the distance between points from the first set to points from the second set. Next, the average distance of all the points in a given portion of blood vessel is calculated, 
providing an estimation of the average diameter of the blood vessel in that portion.

Let $P_{i}$ be the set of points after snakel has finished all its iterations and $Q_{i}$ the set of points after snake 2 has finished all its iterations. For each $Q_{i}$, a line $\mathrm{AB}$ passing through $Q_{i}$ and $Q_{i+1}$ is defined. Then a new line perpendicular to $\mathrm{AB}$ and passing through $Q_{i}$ is calculated.

The distances from points $P_{i-1}, P_{i}$ and $P_{i+1}$ to the perpendicular line are determined. The point that has the minimum distance to the perpendicular line will be taken as the point with shortest distance to $Q_{i}$. The distance between $Q_{i}$ and $P_{i}$ (that can be $P_{i-1}, P_{i}$ or $P_{i+1}$ ) is calculated following the Euclidean distance equation:

$$
d=\sqrt{\left(P_{x i}-Q_{x i}\right)^{2}+\left(P_{y i}-Q_{y i}\right)^{2}}
$$

The distances are stored in a vector, and once all the point to point distances have been calculated the average distance and the typical deviation are determined. To improve the accuracy of the system, the procedure described above is repeated again three more times: with initial line AB passing by $Q_{i}$ and $Q_{i-1}, P_{i}$ and $P_{i+1}$ and $P_{i}$ and $P_{i-1}$ respectively.

This measurement system has proved very efficient computationally (on performing the calculations) and also accurate in the measurements of vessel diameters. More detail can be found in [10].

\section{RESULTS}

The results of the system are presented in a visual form (Fig 10, Fig 11 and Fig 12) showing the vessel boundaries detected (the thickness of these boundaries is exaggerated in the images for visualization purposes). They also include the estimation values for Blood Vessel Diameter calculated by our system compared with results taken from ImageJ [7] which is the method currently in use in blood vessel measurement [4].

\section{1) The system:}

The system was implemented on a PC with a $1.80 \mathrm{GHz}$ AMD Turion64 processor with $2 \mathrm{~GB}$ of RAM. The program was implemented in MATLAB ${ }^{\circ}$ and the results were also graphed with MATLAB ${ }^{\circledR}$.

\section{2) The input data:}

The two images used here were taken from the National Institute of Biomedical Imaging and Bioengineering web site [9]. Both Image 1 (Mouse Brain Blood Vessel) and Image 2 (Mice Alzheimer Blood Vessel) are intra-vital fluorescence microscopy images.

\section{3) Output:}

Visual assessment of the vessel boundaries detected showed precise estimation of their position. To get an idea of the accuracy of the presented approach we compared the diameter estimates generated by our algorithm with manual diameter estimates, using ImageJ [7]. ImageJ is an image processing tool that enables the user to manually draw lines across vessels and records the distance from the start point to the end point.
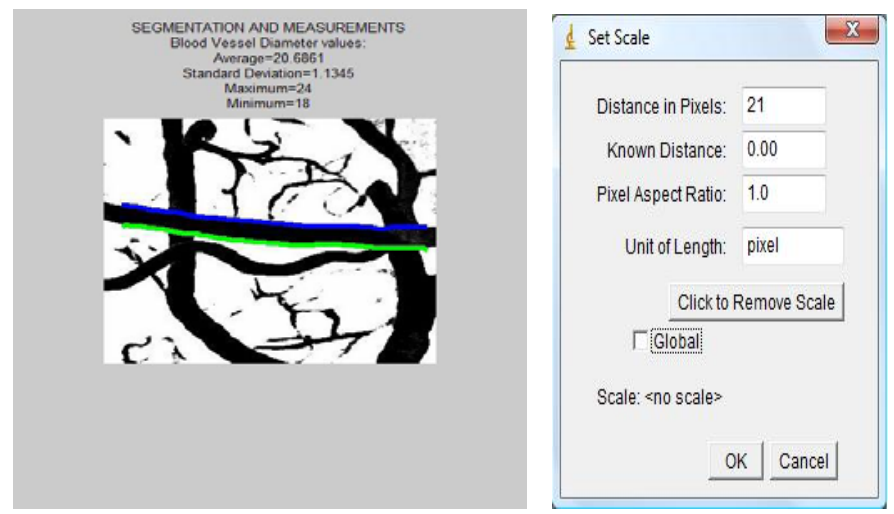

Figure 10. Image 1 Horizontal measurement: System vs ImageJ
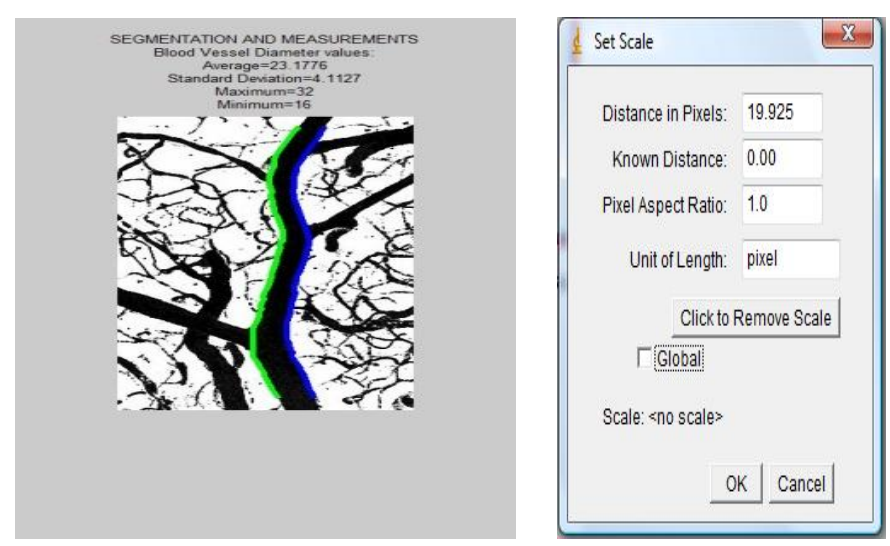

Figure 11. Image 2 Vertical measurement: System vs ImageJ
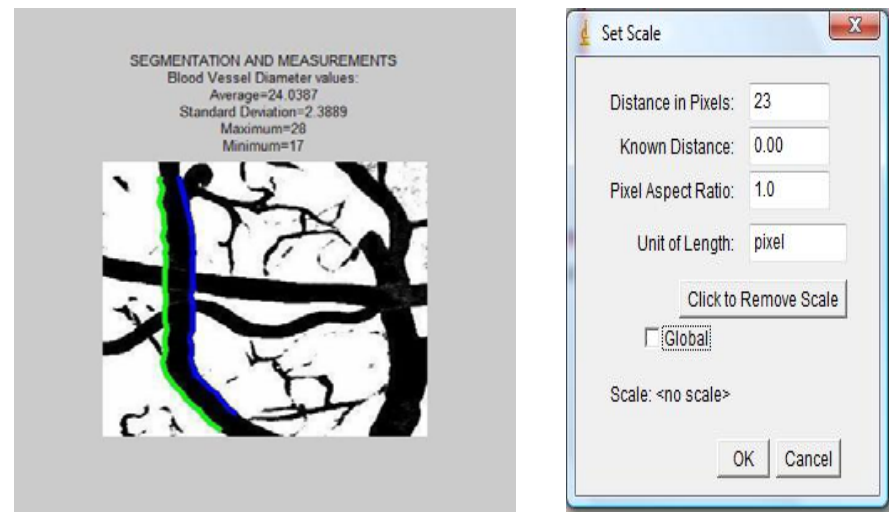

Figure 12. Image 1 Vertical measurement: System vs ImageJ 


\section{CONCLUSIONS}

Segmentation algorithms form the essence for medical image applications such as radiological diagnostic systems, multi-model image registration, visualization, and computer aided surgery. Although many promising techniques and algorithms have been developed, there are still many challenging problems focused on the computation of accurate geometric models of anatomic structures from medical images. This is an open area for more research.

Automatic and semi-automatic approaches to trace vessels share one common problem: their recognition level goes down considerably in regions where vasculature is very dense and/or irregular. Another problem occurs when the noise level in the image increases. Increased noise levels in the image can produce a lower recognition rate which in turns leads to an increase in the time spent on manual post-processing and error correction.

Thus, the decision in this project was to concentrate on designing a reliable blood vessel estimation system that traced the outline of blood vessels from imperfect images and extracted useful information about their dimensions in an automated manner. The segmentation method consisting of two Active Contours (Kass algorithm) proved to be able to detect blood vessel boundaries in a precise way. The measurement method also showed accurate estimation of the blood vessel diameters.

Additional work could be devoted to improve the reliability of the contour detection in some special cases with complex geometry such as bifurcations or severe eccentric stenosis.

Regarding usability, the user interface could be also refined, including a more thorough testing of the system, for it to be used commercially. Following the investigations described here, a future line of work could combine the ChanVese [8] algorithm with the method implemented in this project. Chan-Vese would be used for identifying the whole blood vessel network, while the Kass algorithm refines the areas of interest and the Measurement system provides output accurate results.

In this work, we have achieved our outlined goals of building an automated, portable blood vessel diameter measurement system for use in either diagnosis of diseases or monitoring progress evolution.

\section{ACKNOWLEDGMENTS}

Ana Tizon is grateful to all the people who encouraged her to write this paper. She would also like to acknowledge the reviewers of the first draft and their helpful comments.

\section{REFERENCES}

[1] C. Kirbas, and F. Quek, "A review of vessel extraction techniques and algorithms", ACM Computing Surveys (CSUR), vol.36 no.2, pp. 81-121, June 2004.

[2] D.Nain, A.J. Yezzi and G. Turk, "Vessel segmentation using a shape driven flow", In Proceedings of MICCAI (1), 2004, 51-59

[3] C. Li, C. Kao, J. C. Gore and Z. Ding, "Implicit active contours driven by local binary fitting energy", CVPR 2007.

[4] O. McEnery, L. Lucas, Y. Ma, P. Morrow, C. Mitchell, and K. Saetzler, "A fast, robust and scale-independent approach to estimate vessel diameters in intravital fluorescence microscopy images", $3^{\text {rd }}$ IEEE International Symposium on Biomedical Imaging: Nano to Macro, pp. 101-104, 2006.

[5] M. Kass, A. Witkin, and D. Terzopoulus, "Snakes: Active contour models", Int. J. Comput. Vision 1,4, pp. 321-331, 1988.

[6] A.A. Amini, T.E. Weymouth, R.C. Jain, "Using dynamic programming for solving variational problems in vision", IEEE Transactions on Pattern Analysis and Machine Intelligence, vol. 12, no.9, pp. 855-867, 1990.

[7] W.S. Rasband., "ImageJ", National Institute of Health, Bethesda, Maryland, USA, http://rbs.info.nih.gov/ij/,1997-2005.

[8] T.F. Chan and L.A. Vese, "Active contours without edges", IEEE Transactions on Image Processing, vol. 10, pp. 266-277, 2001.

[9] National Institute of Biomedical Imaging and Bioengineering, http://www.nibib.nih.gov/healthedu/eadvaces/18july07

[10] A. Tizon, "Blood vessel diameter estimation system", Final Project Thesis, Dublin Institute of Technology, Dublin, June 2011. 